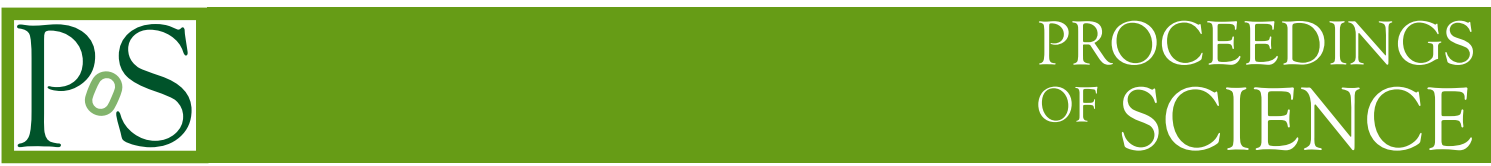

\title{
MEIC Detector and Interaction Region at JLab
}

\section{Zhiwen Zhao'}

Thomas Jefferson National Accelerator Facility

Newport News, Virginia 23606, USA

Old Dominion University

Norfolk, VA 23529, USA

Duke University

Durham, NC 27708, USA

E-mail: zwzhao@jlab.org

\section{JLab MEIC Study Group}

The Electron-Ion Collider (EIC) is envisioned as the next-generation US facility for exploring the strong interaction. The Medium-energy EIC (MEIC) is the first stage of the EIC at Jefferson Lab (JLab). It's aimed at mapping the spin and spatial structure of the quark and gluon sea in the nucleon, understanding the emergence of hadronic matter from color charge, and probing the gluon fields in nuclei. A full-acceptance detector is designed to measure the complete final state. Its interaction region allows spectators tagged with high resolution to catch all nuclear and partonic target fragments. The combination of a high luminosity, polarized lepton and ion beams, and detectors fully integrated with the accelerator will allow MEIC to be a unique opportunity to make breakthroughs in the study of nucleon structure and QCD dynamics.

The XXIII International Workshop on Deep Inelastic Scattering and Related Subjects Dallas, Texas (April 27 - May 1, 2015)

1

Speaker 


\section{Introduction}

The Electron-Ion Collider (EIC) is envisioned as the next-generation US facility for exploring the strong interaction. It's aimed at mapping the spin and spatial structure of the quark and gluon sea in the nucleon, understanding the emergence of hadronic matter from color charge, and probing the gluon fields in nuclei [1]. The Medium-energy Electron Ion Collider (MEIC) at JLab is designed to provide high luminosity and high polarization needed to reach new frontiers in the exploration of nucleon and nuclear structure. The luminosity can range from $10^{33} \mathrm{~cm}^{-2} \mathrm{~s}^{-1}$ to $10^{34} \mathrm{~cm}^{-2} \mathrm{~s}^{-1}$ in a broad range of the center-of-mass (CM) energy. The figure- 8 geometry of the MEIC rings preserves and controls the polarization during the acceleration and collision. A full-acceptance detector is designed to measure the complete final state. The interaction region allows spectators tagged with high resolution to catch all nuclear and partonic target fragments. The detector and interaction region have a coherent design to maximize the physics reach.

\section{Accelerator}

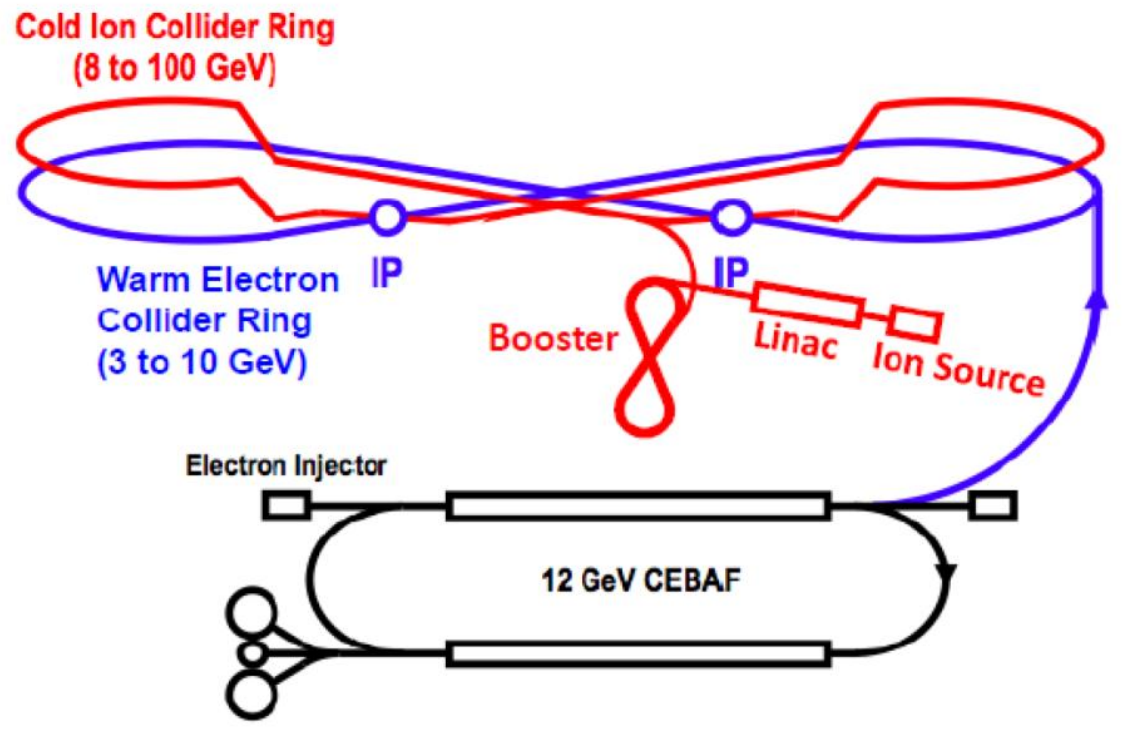

Figure 1: A schematic layout of MEIC complex

The MEIC is designed to be a traditional ring-ring collider [2]. The central part of this facility is two figure- 8 shape collider rings that are vertically stacked and housed in the same underground tunnel, as shown in Figure 1. The figure-8 crossing angle is 81.7 degree, partitioning a collider ring into two arcs and two long straights. The ion beam executes a vertical excursion to the plane of electron ring for a horizontal crossing for electron-ion collisions. Two collider rings have nearly identical circumferences of approximately $2.2 \mathrm{~km}$, and fit well in the Jefferson Lab site.

The electron complex consists of the CEBAF recirculating SRF linac and electron collider ring. CEBAF serves as a full energy injector to the collider ring and provides up to 12 $\mathrm{GeV}$, high repetition rate and high polarization $(>85 \%)$ electron beams. The ion complex 
consists of ion source, linac, booster and collider ring. All required ion species will be generated by two sources, namely, an Atomic Beam Polarized Ion Source (ABPIS) [3] for polarized or unpolarized light ions and an Electron Beam Ion Source (EBIS) [4] or Electron Cyclotron Resonance (ECR) ion source [5] for un-polarized heavy ions up to lead.

The two interaction points (IP) are located when electron beam travels along straight line and ion beam just left the arc to minimize synchrotron radiation from electrons and ions interaction with residual gas in the ion beamline.

\section{Interaction Region and Detector}

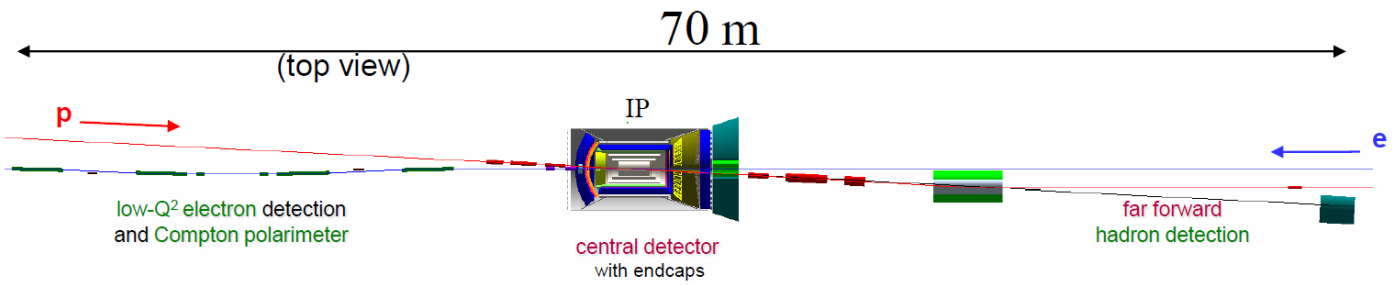

Figure 2: Layout of MEIC interaction region 1 with the full acceptance detector

There are $70 \mathrm{~m}$ space reserved near each IP for its interaction region (IR). Figure 2 show a complete Geant 4 model layout of IR 1, which hosts the full acceptance detector. The ion beam and electron beam collide at IP with $50 \mathrm{mrad}$ crossing angle, which allows the two beamlines to separate quickly when it is away from IP and makes it easy to place beamline magnets and detectors at those often space limited region. Deflected particles will be detected either at the ion downstream side detector or the electron downstream side detector. The central detector will detect the rest of final state particles. Both the ion downstream side detector and the electron downstream side detector take about $30 \mathrm{~m}$ space and the rest $10 \mathrm{~m}$ is used for the central detector. In the next few sections, we will give a brief overview of different sections.

\section{1Ion Downstream Side Detector}

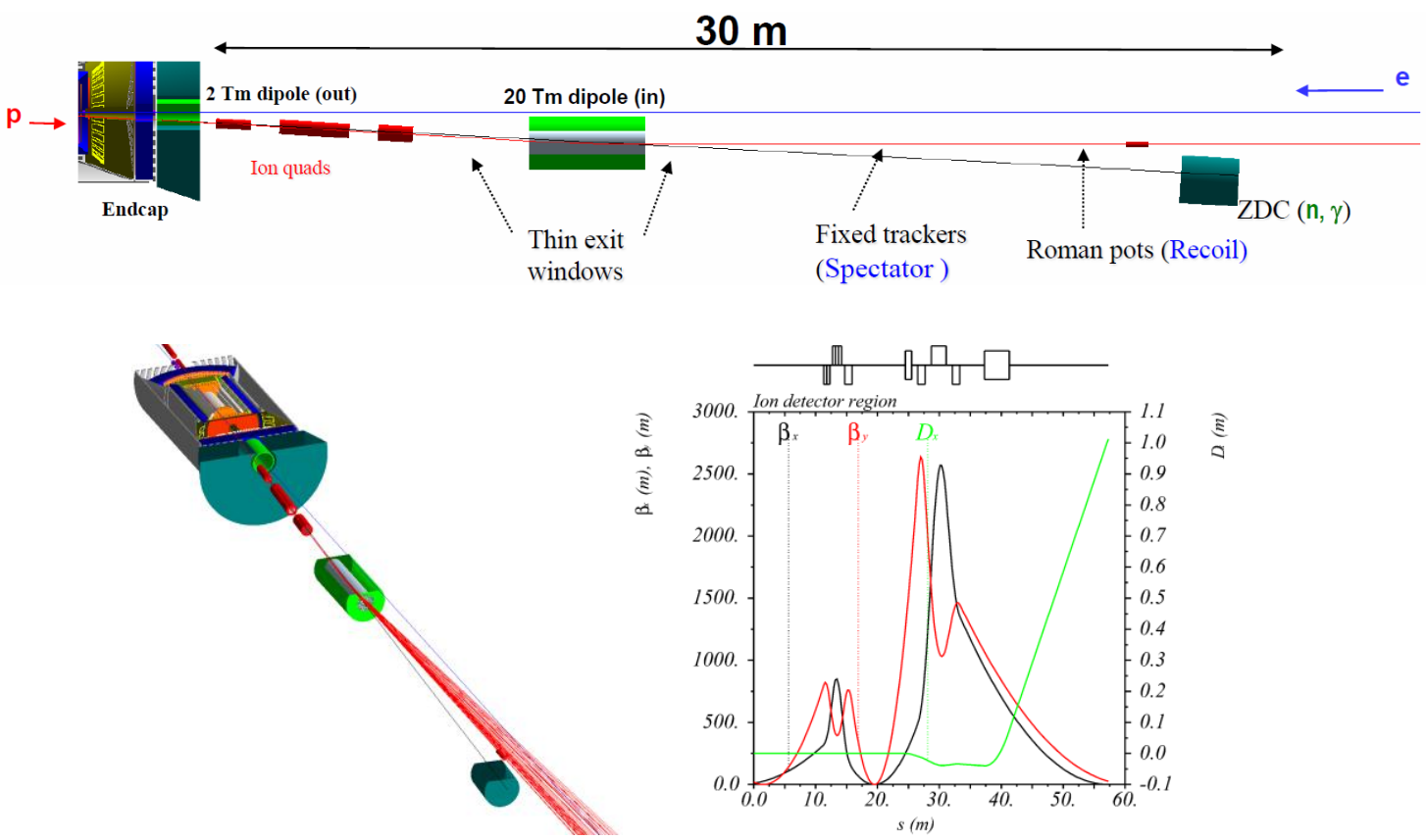

Figure 3: Zoom in on MEIC IR 1 ion downstream side detector 
The ion downstream side detector, as shown in Figure 3, can detect particles within a few degree of the ion beamline. From a few degree to about 1 degree, particles are detected after passing through a $1 \mathrm{~m}$ long $2 \mathrm{Tm}$ out-bending spectrometer dipole in front of the final focusing quads (FFQs). Below 1 degree, they go through the apertures of the FFQs and are detected in a $4 \mathrm{~m}$ space before and a $16 \mathrm{~m}$ space after a second $4 \mathrm{~m}$ long $20 \mathrm{Tm}$ in-bending spectrometer diople. Spectator neutrons follow the ion beam direction at IP have clearance to pass through all beamline magnets and are detected by a zero degree calorimeter at the very end when it is well separated from the beamline. Spectator protons and slightly deflected protons are detected by a movable Roman pot to get close to the beamline. The optics is optimized to maintain small aperture ( $\beta$ ) at IP and large dispersion (D) in the $16 \mathrm{~m}$ long drift space to allow a set of tracking and particle identification detectors to detect particles with wide range of kinematics.

\section{2Electron Downstream Side Detector}
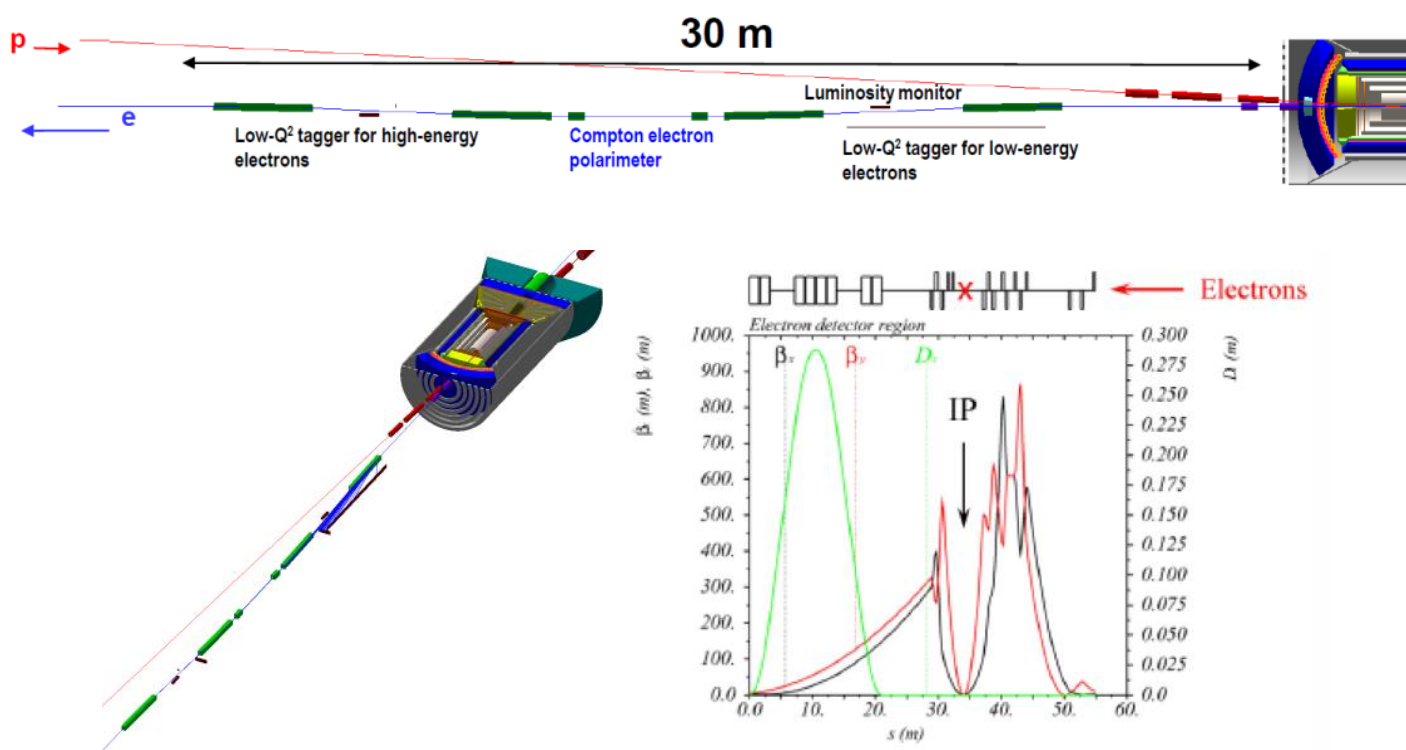

Figure 4: Zoom in on MEIC IR 1 electron downstream side detector

Figure 4 shows the electron downstream side detector. Large aperature FFQs allow electrons scattered at a few degrees to pass through. Then they are detected by a chicane of 4 dipoles with various detectors, which is easier to see in Figure 5.

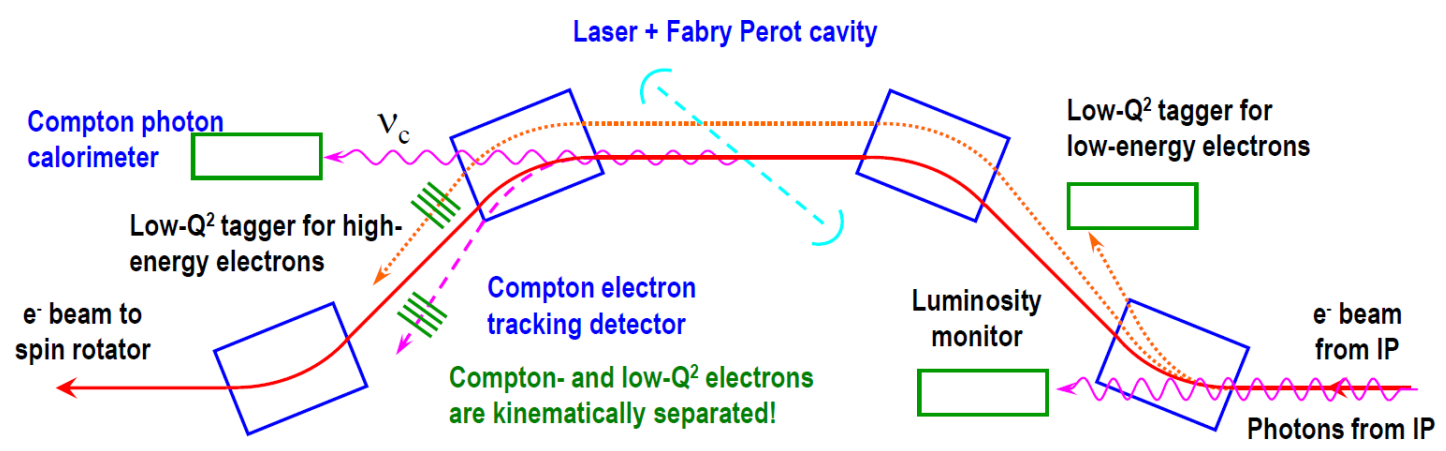


Figure 5: A schematic layout of MEIC IR1 electron downstream chicane, which consists of 4 dipoles and various detectors.

After 1st dipole, there are the luminosity monitor and the low $\mathrm{Q}^{2}$ tagger for low energy electrons. The low $\mathrm{Q}^{2}$ tagger for high energy electrons is after 3rd dipole. Between the 2 nd and 4th dipoles, where the optics has a nice plateau of the dispersion (D), it is the place of the electron polarimeter by measuring the Compton reaction between electrons and photons from a laser. This kind of nonintrusive and always-on electron polarimeter has been successfully used at Jeffersion Lab and is cricual for physics programs requiring high precision polarization measurement.

\subsection{Central Detector}

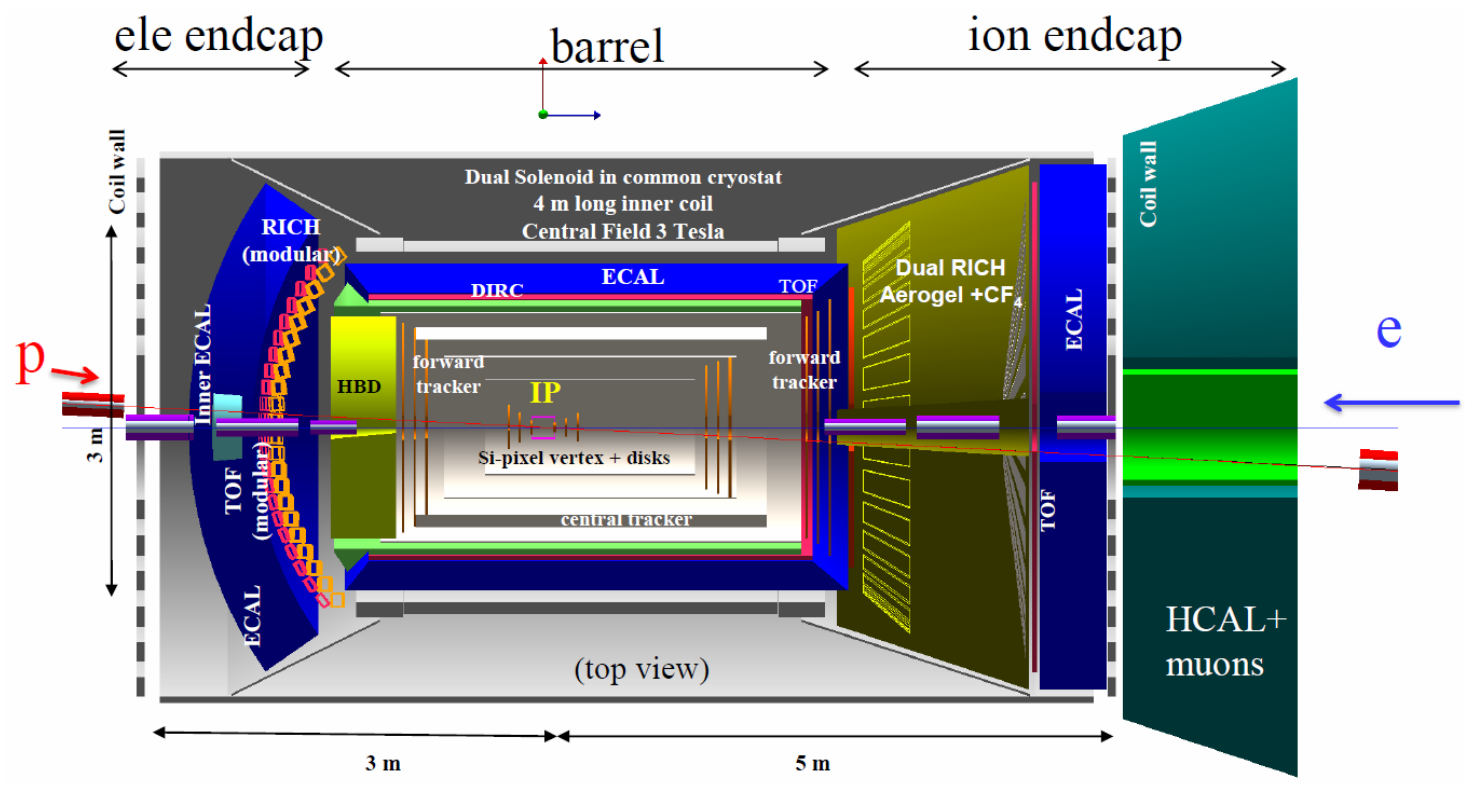

Figure 6: Zoom in on MEIC IR 1 central detector

The central detector is based on a dual solenoid design. The inner and outer solenoid coils run currents in the directions opposite to each other and thus effectively cancel the magnetic field outside. With the help of two endcap coil walls, this design elminates the need to have bulky iron as flux return, therefore leaves a lot of space for detectors.

As shown in Figure 6, the central detector can be roughly seperated into three sections, namely, barrel, ion endcap, electron encap. Near the IP, there is a set of high resolution trackers to provide good vertex determination. Going outside, a few grouped thin layer tracks cover the barrel and both endcaps. The barrel detectors include DIRC, time of flight (TOF) and scintilation fiber based electromagnetic calorimeter (ECAL). The ion endcap has a dual radiator RICH, a layer of TOF and a shashlik ECAL. The hadron calorimeter (HCAL) and possible muon detector are outside of the endcap and around the 1st ion downstream dipole. In the electron endcap, we have a projective design to align the modular RICH, modular TOF and special shaped shashlik ECAL while the hadron blind detector (HBD) provides electron and pion seperation. 


\section{Summary}

We have given a brief introduction to MEIC. Its accelerator has the figure 8 design to preserve and control polarization. Its fully integrated interaction region and full acceptance detector allow the measument of the complete final state. These features enable MEIC to be a unique opportunity to make breakthroughs in the study of nucleon structure and QCD dynamics.

\section{References}

[1] A. Accardi et al., EIC white paper, (2012) [arXiv:1212.1701]

[2] S. Abeyratne et al., MEIC Design Summary, (2015) [arXiv:1504.07961]

[3] T. Clegg et al., NIM A, Vol.357, Issues 2-3, p.200-211 (1995)

[4] J. Alessi et al., Proc. of the LINA-2006, p.385 (2006).

[5] R. Geller, Annual Review of Nuclear and Particle Science, Vol.40, 15-44 (1990) 